\title{
Management for Preventing Ventilator-Associated Pneumonia at ICU Panti Rapih Hospital: A Clinical Case Study
}

\author{
Siwi Ikaristi Maria Theresia ${ }^{1}$ \\ ${ }^{1}$ Panti Rapih Nursing Academy, Indonesia \\ Corresponding author: siwi_theresia@yahoo.co.id
}

\begin{abstract}
Background: Ventilator-Associated Pneumonia (VAP) is one kind of nosocomial infection that happens to patients who use mechanical ventilator and tracheostomy. Patients who are hospitalized in Intensive Care Unit (ICU) would have high risk on suffering from Pneumonia.

Objective: This paper aims to describe the interventions of nurses and to give recommendations for managing the VAP prevention using case study method.

Methods: The data were collected from a patient who used mechanical ventilator and continued by observing and interviewing the nurses who took care of the patient.

Results: The development of late onset of VAP was noticed after 96 hours. The observation result showed that there were fungi appeared after conducting a sputum culture from the patient. There was an increase of White Blood Cells on 72 hours after the intubation and the temperature increased until $37^{\circ} \mathrm{C}$ while the patient was using ventilator. Unfortunately, the nurses were not aware of the tendency of late onset VAP development in the patient.

Discussion: Fungi are microorganism which causes VAP. The fungi were not proliferating because the patient had received early antibiotics for treatment. Early administration of antibiotics will prevent the development of VAP, but late VAP is associated with antibiotic-resistant organism. The significant increase of WBC count and the tendency of temperature while the patient was using ventilator were signs for developing infection. VAP can be prevented by conducting good oral hygiene every 2 hours using chlorhexidine $0.12 \%$ and using orogastric tube and ETT with sub glottic port to patients who use mechanical ventilator. Furthermore, positioning the patient's head with $35^{\circ}-45^{\circ}$ bed elevation is intended to prevent aspiration as well as checking adequate endotracheal tube cuff of at least $20 \mathrm{~cm} \mathrm{H} 2$.

Conclusion: Awareness, responsibility, knowledge, skills of nurses are basis for infection control especially for preventing VAP incidents.
\end{abstract}

Keywords: Ventilator Associated Pneumonia (VAP), Intensive Care Unit (ICU), management of prevention, nurses. 


\section{BACKGROUND}

Nosocomial infection is a wide area that brings an effect to patients on mortality, morbidity, length of stay and excess healthcare costs (Koenig \& Truwit, 2006). Ventilator-Associated Pneumonia (VAP) is one kind of nosocomial infections that happens to patients who use mechanical ventilator and tracheostomy. Patients who are hospitalized in Intensive Care Unit (ICU) would have high risk on suffering from Pneumonia. According to Inweregbu, Dave, \& Pittard (2005) the highest prevalence of nosocomial infection in hospital setting was in ICU (20.6\%) which was caused by mechanical ventilation.

Ventilator-associated pneumonia (VAP) usually occurs 48-72 hours or thereafter following endotracheal intubation, characterized by the presence of a new or progressive infiltrate, signs of systemic infection (fever, altered white blood cell count), changes in sputum characteristics, and detection of a causative agent (Kalanuria, Zai, \& Mirski, 2014). VAP rates range from 1.2 to 8.5 per 1,000 ventilator days and are reliant on the definition used for diagnosis. Risk for VAP is greatest during the first 5 days of mechanical ventilation (3\%) with the mean duration between intubation and development of VAP being 3.3 days (Rello et al, 2002). This risk declines to $2 \% /$ day between days 5 to 10 of ventilation, and $1 \%$ /day thereafter (Cook et al., 1998). Over the years, the attributable risk of death has decreased and is more recently estimated at 9-13 $\%$ largely because of implementation of preventive strategies (Melsen et al., 2013).

Mechanical ventilation uses invasive procedures and causes immune compromise status. Environment and health care workers have some roles in preventing this infection. Hence, they must be aware and responsible to prevent the growth of the VAP (Muscedere et al., 2008). Furthermore, interventions of ICU nurses to prevent VAP incident becomes an important part because during 24 hours they always take care of the patients. The nurses should have knowledge and skill on how to manage the prevention of VAP in the clinical practice. The case study method could be used to evaluate and develop interventions to prevent VAP in order to improve quality of the health care.

Panti Rapih Hospital is a referral hospital in Yogyakarta which has well developed ICU ward. The hospital has eight mechanical ventilators to help patients survive from respiratory failures. The patients are expected to develop VAP if they use the medical devices such as mechanical ventilator and tracheostomy. Unfortunately, the hospital does not have data containing some detailed information about patients suffering from VAP because the hospital is concentrating on VAP preventing management. Therefore, in order to describe the management for preventing VAP, a clinical case study was conducted to find out the strategies applied by the hospital to prevent VAP in ICU.

\section{OBJECTIVE}

This paper aims to describe the interventions of nurses and to give recommendations for managing the VAP prevention using case study method.

\section{METHODS}

This study employed case study research method. The study described the management of Ventilator-Associated Pneumonia prevention in ICU. The study took a patient who 
used mechanical ventilator as a case to be studied. The researcher started collecting data from a patient and ICU nurses by physical assessments, observation, interview and analyzing the medical record of the patient. Furthermore, the data were analyzed by comparing and contrasting them with the concepts of VAP.

\section{RESULT}

\section{Case History}

Mrs. S is a 68 years old female with a medical history of ileus obstruction. On May 18, 2013 she underwent an open laparatomy. In the preoperative period, chest X-Ray result showed she had normal lungs. During the surgery, she developed new onset Atrial Fibrillation and respiratory failure. In order to handle the problem, the doctor intubated her and recommended to use mechanical ventilator. Mode and setting of the ventilator were SIMV +PS, Fi $\mathrm{O}_{2} 35 \%$, SP O $299 \%$ - 96\%. She was then transferred to ICU.

In the ICU, she was intubated in conscious level, used nasogastric tube, catheter urine and two infusion lines. The patient got Merofen antibiotic 1 gram two times a day at $08.00 \mathrm{am}$ and $08.00 \mathrm{pm}$ immediately after intubation. Two days after intubation, her white blood cell (WBC) was checked and the result was normal $\left(4.7 \times 10^{3}\right)$. Furthermore, on May 20, the patient's chest was rechecked using X-Ray and the result was clear. Also, her sputum culture and WBC were rechecked. Fungi were found in the sputum culture but they were not proliferating. The result of the WBC was high $\left(14.6 \times 10^{3}\right)$. While the patient was using ventilator, nurses suctioned her to clear her airway from fluids and sputum because she often had difficulties on swallowing and coughing. ICU nurses always used closed suction for suctioning. Patient's sputum looked cleaner.

Furthermore, while the patient was using mechanical ventilator, nurses checked her body temperature every 2 hours and the result was about $36-37^{\circ} \mathrm{C}$ (normal body temperature under treatment of the ventilator). Besides, ICU nurses always did oral hygiene and bed bathed the patient every morning and afternoon. Oral hygiene was performed using cotton and chlorhexidine. Meanwhile, nurses bathed the patient using water, soap and basin. The patient was sleeping with 35-45 degrees head of bed elevation in order to avoid aspiration. On May 22, ICU nurses did weaning process because her respiratory looked adequate and had normal heart beats. During the weaning process, nurses always observed on adequate respiratory, minimum secretion of cough, heart beats and oxygen saturation. Besides, she had an adequate conscious level and then the ventilator was removed. The patient used mechanical ventilator for 4 days.

Table 1. Data observation of the patient in the ICU on May 18-22, 2013

\begin{tabular}{llllll}
\hline Date & $\mathbf{1 8}$ & $\mathbf{1 9}$ & $\mathbf{2 0}$ & $\mathbf{2 1}$ & $\mathbf{2 2}$ \\
\hline Temperature ${ }^{\mathrm{O}} \mathrm{C}$ & 36 & 36.5 & 36.8 & 37 & 36.8 \\
WBC & $4.7 \times 10^{3}$ & & $14.6 \times 10^{3}$ & & \\
Sputum & White & White & White & White & Purulent \\
& clear & clear & $\begin{array}{l}\text { clear } \\
\text { clear }\end{array}$ & \\
Lungs sound & clear & clear & clear & clear & clear \\
\hline
\end{tabular}


Meanwhile, chlohexidine and alcohol for hands rub were placed in every room. By observing the behavior of ICU nurses and other healthcare employees that took care of Mrs. S, they always washed their hands before and after doing activities to the patient. The nurses also always wore masks to take care of the patient. Unfortunately, the nurses rarely maintained adequate endotracheal tube cuff of at least $20 \mathrm{~cm} \mathrm{H}_{2}$, whereas it was important to prevent aspiration.

Furthermore, in order to record the whole information related to the patients, the nurses always checked the patient's vital signs such as checking temperature, blood pressure, pulse, oxygen saturation, respiratory rate, conscious level and administered medications as prescribed. Unfortunately, during taking care of Mrs. S, the nurses never wrote the result of lung sounds and sputum characteristic on chart. At the end of the clinical case study, a primary nurse of the patient said that Mrs. A was not suffering from VAP, although the sputum culture was found fungi but the chest X-ray did not show new infiltrate, the sputum was white clear and the temperature was normal.

\section{DISCUSSION}

After conducting an analysis based on the case and concept of VAP, the actual fact showed that the patient suffered from late onset VAP. Unfortunately, the ICU nurses concluded that the patient was not suffering from VAP. However, the patient had an indication of infection tendency after 96 hours. Amanullah (2013) stated that VAP is a nosocomial infection that grows for 48 hours or more after a mechanical ventilator is given by means of an endotracheal tube or tracheostomy. The VAP incidents occurred to $28 \%$ of patients who receiving mechanical ventilator in ICU. Supported by Augustyn (2007), the etiology of VAP is divided into two types; those are early VAP and late VAP. Early VAP occurs 48 to 96 hours after intubation and late VAP occurs after 96 hours after intubation. Early VAP is associated with antibiotic-susceptible organism and late VAP is associated with antibiotic-resistant organisms. The assumption of developing late onset VAP was based on two major data which were the finding of some fungi after the sputum culture and the use of medical devices of the patient.

Some fungi were found after performing the sputum culture, although it was nonspecific fungi and the fungi was not breeding. Fungi are microorganism which cause VAP, although the fungi are not the primary cause of VAP. The fungi were not proliferating because the patient had received early antibiotic for treatment. The patient received Merofen antibiotic 1 gram for twice a day from her doctor and the nurses always administered the medicine as appropriate procedure for administering medication. The antibiotic was appropriate to treat patient with VAP. Early administration of antibiotic will prevent for developing VAP, but late VAP is associated with antibiotic-resistant organism. Safdar, Crnich, \& Maki (2005) stated common causes of VAP are bacteria species including gram negative organism, gram positive organism and fungi. The most common cause of VAP is gram-negative bacteria. Meanwhile, American Thoracic Society and Infection Disease of America states the criteria for diagnosis of VAP were new infiltrate on chest radiographs after 48-72 hours of endotracheal intubation and presence of at least 2 of following: fever (temperature is more than $38^{\circ} \mathrm{C}$ ) abnormal $\mathrm{WBC}$ count and purulent respiratory tract secretion and microorganisms in cultures sample (Su et al., 2012). It was significant for increasing 
WBC count (14.6x103) of the patient on 72 hours after intubation. Increasing WBC count is an indication of infection. The tendency of temperature data increased until $37^{\circ}$ $\mathrm{C}$ while the patient was using ventilator, because the tendency of patient's temperature increased as it was seen from the significant sign. Besides, the sputum was purulent after 96 hours was a sign for developing late onset of VAP.

The patient had a high possibility to suffer from VAP because she used medical devices including mechanical ventilator, endotracheal tube, nasogastric tube, catheter, and two infusion lines. According to Safdar et al. (2005), the pathophysiology of VAP results an invasion of microorganisms to lower respiratory track and lung parenchyma. There are some routes of VAP development including aspiration of microorganism secretion from oropharynx directly and reflux from stomach into oropharynx then into the lower respiratory tract. Besides, the route of development VAP is by direct extension of a contiguous infection such as a pleural space infection, by inhalation of contaminated air or medical aerosol and by hematogenous carriage of microorganism to the lungs from remote sites of local infection such as vascular or urinary tract-related bloodstream infection. Supported by Augustyn (2007), the late-onset of VAP occurred after 96 hours after intubation. The risk factors for VAP are host related, devices related and personnel related. Host related includes medical condition, immunosuppression, chronic obstruction lung disease, adult respiratory distress syndrome, patient's body position, level of consciousness, number of intubations and medications. Device related includes endotracheal tube, ventilator circuit and nasogastric or orogastric tubes. Personal related includes improper hand washing, failure to change gloves between contact to patients and not wearing personal protective equipment when antibiotic resistant bacteria have been identified.

Meanwhile, healthcare team of the patient which included nurses and physicians showed the management for preventing VAP. The following activities were the interventions of nurses and doctors to prevent VAP. First, the healthcare team washed their hand using chlorhexidine disinfectant before and after doing activities to the patient, wearing gloves and masks during the interaction with the patient. Second, positioning patient's head with the bed elevation of $35^{\circ}-45^{\circ}$ was intended to prevent aspiration but the nurses rarely maintained adequate endotracheal tube cuff of at least 20 $\mathrm{cm} \mathrm{H}_{2}$. Third, performing oral hygiene used chlorhexidine $0.12 \%$ twice a day. Fourth, suctioning of fluid and sputum used closed suction system. Fifth, nasogastric tube was used to give enteral nutrition and to avoid abdominal distention. Last, ICU nurses often wrote their activities to the patient's chart such as checking temperature, blood pressure, pulse, oxygen saturation, respiratory rate, conscious level and administering medications as prescribed.

Management for preventing VAP has becomes an important part of nosocomial infection control. Health care workers should be aware and do standardized operational procedure for preventing the nosocomial infection. According to Sedwick et al. (2012), VAP bundles can be an effective way to prevent VAP. The VAP bundles includes daily interruptions of sedation and readiness extubation, peptic ulcer prophylaxis, deep vein thrombosis prophylaxis, elevate the head of the bed more than $30^{\circ}$ (semi recumbent position) to avoid risk factor of aspiration and pneumonia, daily oral care with 
chlorhexidine $0.12 \%$ every two hours because dental plague and accumulation of salivary can provide respiratory pathogen such as MRSA and Pseudomonas aeruginosa, hand washing protocol and sub glottic suctioning.

Augustyn (2007) supported the management for preventing VAP using two strategies which include prevention of colonization and prevention of aspiration. There are some ways that can be used to prevent colonization. The first way uses oral decontamination and the second one uses stress ulcer prophylaxis. The third way is turning patients at least every 2 hours. The last one is adjusting the ventilator no more than 48 hours. To prevent aspiration, a nurse can adjust the position of the head of bed into $>30^{\circ}$, minimize the use of narcotic and sedative agent, do oropharyngeal suctioning, and use endotracheal tubes that have continuous sub glottic ports. Monitoring gastric residual for over distention can also become a useful way to prevent aspiration which will be continued by maintaining adequate endotracheal tube cuff of at least $20 \mathrm{~cm} \mathrm{H}_{2}$. Meanwhile, Bouadma et al (2010) recommended to use orogastric tube than nasogastric tube in order to reduce the frequency of sinusitis and possibly VAP. Siempos, Vardakas, \& Falagas (2008) also recommended to use closed suction system than open suction system because the closed suction system has an advantage to decrease environmental contamination from spread of multidrug resistant pathogen rather than open suction system.

\section{CONCLUSION}

Ventilator Associated Pneumonia is a serious problem of nosocomial infection which happens in ICU setting because VAP brings effect on mortality, morbidity, cost and length of hospital stay. Awareness, responsibility, knowledge, and skills of nurses are the basis for infection control especially for preventing VAP incident. By studying clinical case of management in preventing VAP, it could help nursing staffs to develop their interventions and to evaluate their programs in preventing VAP. Some recommendations for ICU nurses from the findings are considering the increase of WBC count of patients who used medical devices, considering the tendency on increasing temperature data day by day, collaborating with physician for recheck laboratory and chest $\mathrm{x}$-ray when some abnormal conditions were found, performing an oral hygiene every 2 hours using chlorhexidine $0,12 \%$, considering the use of orogastric tube and ETT with sub glottic port to patients who use mechanical ventilator, and maintaining endotracheal tube cuff of at least $20 \mathrm{~cm} \mathrm{H} 2$ to prevent aspiration every day.

\section{REFERENCES}

Amanullah, S.(2013).Ventilator-Associated Pneumonia Overview of Nosocomial Infection. Retrieved July 12, 2013, from Medscape Reference: http://emedicine.medscape.com/article/304836-overview\#showall

Augustyn, B. (2007). Ventilator-associated pnuemonia: risk factors and prevention. Critical Care Nurse, 27(4), 32-39

Bouadma, L., Mourvillier, B., Deiler V., Corre B L., Lolom, I Regnier, B., Wolff M and Lucet, J C. (2010). A multifaceted program to prevent ventilator-associated pneuonia: impact on compliance with preventive measure. Critical Care Medicine, 38(3), 789-796. 
Cook, D. J.,Walter, S. D., Cook, R. J...et al. (1998). Incidence of and Risk Factors for Ventilator-Associated Pneumonia in Critically Ill Patients. Annals of Internal Medicine, 129(6), 433-440.

Inweregbu, K., Dave, J and Pittard, A. (2005). Nosocomial infections. Continuing Education in Anaesthesia, Critical Care \& Pain, 5(1), 14-17.

Kalanuria, A. A., Zai, W., \& Mirski, M. (2014). Ventilator-associated pneumonia in the ICU. Critical care, 18, 20-29.

Koenig, S. M \& Truwit, J. D. (2006). Ventilator-Associated Pneumonia: Diagnosis, Treatment, and Prevention. Clinical Microbiology Reviews, 19(4): 637-657.

Melsen, W. G., Rovers, M. M., Groenwold, R. H...et al. (2013). Attributable mortality of ventilator-associated pneumonia: A meta-analysis of individual patient data from randomised prevention studies. The Lancet Infectious Disease, 13(8), 665671.

Rello, J., Ollendorf, D. A., Oster, G....et al. (2002). Epidemiology and Outcomes of Ventilator-Associated Pneumonia in a Large US Database. Chest Journal, 122(6), 2115-2121.

Safdar, N., Crnich, C J and Maki, D G. (2005). The pathogenesis of VentilatorAssociated Pneumonia: Its relevance of effective strategies for prevention. Respiratory Care, 50(6), 725-741.

Sedwick, M B., Lance-Smith, M., Reeder, S J and Nardi, J. (2012). Using evidancebased practice to prevent ventilator associated pneumonia. Critical Care Nurse, 32(4), 41-50.

Siempos, I I, Vardakas, K Z., and Falagas, M E. (2008). Closed tracheal suction system for prevention of ventilator-associated pneumonia. British Journal of Anaesthesia, 100(3), 299-306

Su, L., Meng, K., Zhang, X., Wang, H., Yan, P., Jia, Y., Feng, D and Xie, L. (2012). Diagnosing ventilator-assosiated pneumonia in critically ill patients with sepsis. American Journal of Critical Care, 21(6), 110-119. 